\title{
Curing advanced melanoma by 2025
}

Dummer, Reinhard ; Goldinger, Simone M ; Paulitschke, Verena ; Levesque, Mitchell P

\begin{abstract}
PURPOSE OF REVIEW To outline the most urgent challenges in the management of advanced melanoma. RECENT FINDINGS Considerable progress in targeted and immunotherapy of advanced melanoma has opened a perspective for a cure if all molecular and medical information is integrated in a rational precision treatment algorithm. SUMMARY Bioinformatics and system biology approaches will be needed to deal with omics databases. The support of patient advocacy groups may help to increase the acceptance of large scale, routine biobanking.
\end{abstract}

DOI: https://doi.org/10.1097/CCO.0000000000000168

Posted at the Zurich Open Repository and Archive, University of Zurich ZORA URL: https://doi.org/10.5167/uzh-109846

Journal Article

Published Version

Originally published at:

Dummer, Reinhard; Goldinger, Simone M; Paulitschke, Verena; Levesque, Mitchell P (2015). Curing advanced melanoma by 2025. Current Opinion in Oncology, 27(2):125-127.

DOI: https://doi.org/10.1097/CCO.0000000000000168 


\title{
Curing advanced melanoma by 2025
}

\author{
Reinhard Dummer, Simone M. Goldinger, Verena Paulitschke, and \\ Mitchell P. Levesque
}

\begin{abstract}
Purpose of review
To outline the most urgent challenges in the management of advanced melanoma.
\end{abstract}

\begin{abstract}
Recent findings
Considerable progress in targeted and immunotherapy of advanced melanoma has opened a perspective for a cure if all molecular and medical information is integrated in a rational precision treatment algorithm.
\end{abstract}

\section{Summary}

Bioinformatics and system biology approaches will be needed to deal with omics databases. The support of patient advocacy groups may help to increase the acceptance of large scale, routine biobanking.

\section{Keywords}

BRAF, checkpoint inhibitor, kinase inhibitor, MEK, melanoma

After decades of stagnation, targeted therapy and immunotherapy have dramatically improved the outcome of metastatic melanoma treatment. The combination of BRAF and MEK inhibitors achieves response rates of $70 \%$, with progression-free survival of approximately 10 months. These treatments have further enhanced the overall survival in comparison to monotherapy with a BRAF inhibitor [1-3]. However, resistance is still problematic [4]. In this issue, McArthur and colleagues discuss the mode of action and perspective of cyclin-dependent kinase inhibitors. There is evidence that resistance during the inhibition of the mitogen-activated protein kinase pathway can be delayed by the combination of BRAF-MEK inhibitors with cyclin-dependent kinase inhibitors $[5,6]$. If the tolerability of these triple therapies will be as acceptable as the combination therapy, we might expect additional benefit for this approach.

Monoclonal antibodies interfering with checkpoint inhibitors on the surface of T cells, dendritic cells, or tumor cells such as anti-cytotoxic T-lymphocyte-associated protein-4 antibodies and antiprogrammed cell death protein 1 (PD1) antibodies have revolutionized immunotherapy for melanoma and are currently evolving into promising approaches for other malignancies.

For decades, there has been an ongoing interest in immunotherapy based on the clinical observations of spontaneous regressions associated with T-cell infiltrates. However, there were a number of very frustrating clinical trials using vaccines, dendritic cell approaches, or cytokine therapies [7].
Very recently, prospective randomized controlled trials demonstrated that immunotherapy with the two anti-PD1 antibodies pembrolizumab and nivolumab has higher response rates, longer response durations, and less severe adverse events than the chemotherapy used in this clinical situation [8]. There are signals from phase I and II clinical trials that the efficacy of anti-PD1 antibodies can be further increased by the addition of ipilimumab. If the early data of a 1-year survival rate of more than $80 \%$ and the 2 -year survival rate of more than $70 \%$ can be confirmed in prospective randomized trials, then the combination of the two checkpoint inhibitors ipilimumab and nivolumab will be a very promising first-line therapy for advanced metastatic melanoma. However, in contrast to anti-PD1 monotherapy, this combined immunotherapy approach is associated with a number of severe typically immune-related adverse reactions that can become life threatening if not recognized and not treated early with rigorous immunosuppression using corticosteroids and TNF-alpha antagonists [9].

The availability of different promising therapeutic approaches demands an intensive search

Department of Dermatology, University Hospital Zurich, Zurich, Switzerland

Correspondence to Reinhard Dummer, MD, Vice Chairman, Department of Dermatology, University Hospital Zurich, Gloriastrasse 31, 8091 Zurich, Switzerland. Tel: +41 4425525 07; fax: +41 4425525 07; e-mail: reinhard.dummer@usz.ch

Curr Opin Oncol 2015, 27:125-127

DOI:10.1097/CCO.0000000000000168 
for predictive biomarkers that better stratify patients for the respective treatment. For targeted therapy, the presence of a BRAF or NRAS mutation gives the rational basis for the first-treatment interventions using a BRAF and MEK inhibitor, combination, or a MEK inhibitor monotherapy. However, in order to optimize therapy and to prevent early resistance, additional mutations might be considered. In the near future, at least targeted deep exon sequencing will be available for the majority of patients. Clinicians will have to learn how to integrate these molecular data into treatment decisions.

Predictive markers for immunotherapy are also on the horizon, but still need confirmation in clinical trials. Obviously, the situation for immunotherapy will be even more complex. We expect that no single parameter will predict immune susceptibility, but rather a matrix of clinical and immunological factors in the tumor microenvironment or present systemically may provide the strongest predictive power of a patient's potential response to immunotherapy. In this context, integrating immune therapy markers and molecular features, such as neo antigens, will be critical [10].

As the understanding of melanoma progression becomes increasingly more complex, omics databases will play a more important role in patient stratification strategies and future scientific endeavors to develop novel therapies. Thus, these challenges will require intensive cooperation with bioinformaticians and system biology experts who know how to integrate systems-level measurements of genetic and epigenetic alterations, transcriptional profiles, and proteomics data into predictive or targetable pathways. This comprehensive molecular understanding must result in a more complex description of the patient's tumor that gives a rational basis for individual treatment decisions facilitating a multidimensional precision medicine approach.

Obviously, the decision matrix must be based on the self-learning programs that integrate the outcomes of earlier experience into the decisionmaking process in order to provide clinicians with the best possible options for their patients.

As many melanoma patients whose primary tumors are excised today will metastasize only in 5-20 years, the primary tumors collected today are already of tremendous relevance for the individual patient. Therefore, all academic centers should initiate biobank approaches and try to collect primary tumors for these centralized biobanks. Unfortunately, primary tumors today are often removed by private practitioners or surgeons and sent to private pathology laboratories that do not preserve the tumor tissue longer than legally necessary. Therefore, we have initiated a biobank project at the University Hospital Zurich that is able to collect primary tumors in cooperation with private dermatopathology laboratories in the area.

Patient advocacy groups also play an important role in bringing more effective treatments to the clinic. Melanoma survivors are founding increasingly stronger and better organized advocacy groups, and the improved outcomes of newly available therapies will foster the growth of these groups. However, the socioeconomic and political environment regulating clinical research and biobanking is challenging. Academic physicians at university clinics face more and more legal hurdles that slow down their work. For example, even small retrospective studies now require specific ethical approval, which leads to increased workloads for both researchers and ethical committees and delays the pace of ground-breaking research. Although these laws are an important instrument to protect patients' rights, a better balance must be struck that both ensures those rights and encourages the development of the next therapy that may save thousands of lives. When patients understand that they may directly benefit from clinical and translational cancer research, they are eager to cooperate and to support clinical research teams. However, there is a need for a constant exchange of information and ideas between patient advocacy groups and clinical researchers as well as translational cancer biologists. In addition, patient-related outcome such as the quality of life will get more and more important. In contrast to the earlier studies, quality-of-life projects are now accompanying many large phase III clinical trials [11].

In summary, we feel lucky to have everything in our hands: new powerful drugs and high-throughput molecular tools to better understand tumor tissues. Sophisticated immunology research has highlighted the essential checkpoints and targets for immunotherapy, and bioinformaticians and computational biologists can help to model these huge datasets. We must convince our patients and regulating authorities that biobanking and systemslevel analyses of larger patient cohorts during targeted approaches and immune therapy can create rational algorithms that will optimize treatment success and improve the lives of our patients.

\section{Acknowledgements}

None.

\section{Financial support and sponsorship}

This work was supported in part by the $G+B$ Schwyzer Stiftung, the Skin Cancer Research Foundation (Verein für Hautkrebsforschung), and the Stiftung Empiris. 


\section{Conflicts of interest}

None.

\section{REFERENCES}

1. Larkin J, Ascierto PA, Dreno B, et al. Combined vemurafenib and cobimetinib in BRAF-mutated melanoma. N Engl J Med 2014; 371:1867-1876.

2. Long GV, Stroyakovskiy D, Gogas $H$, et al. Combined BRAF and MEK inhibition versus BRAF inhibition alone in melanoma. N Engl J Med 2014; 371:1877-1888.

3. Robert C, Karaszewska B, Schachter J, et al. Improved overall survival in melanoma with combined dabrafenib and trametinib. N Engl J Med 2015; 372:30-39.

4. Dummer R, Flaherty KT. Resistance patterns with tyrosine kinase inhibitors in melanoma: new insights. Curr Opin Oncol 2012; 24:150-154.
5. Grimaldi AM, Simeone E, Ascierto PA. The role of MEK inhibitors in the treatment of metastatic melanoma. Curr Opin Oncol 2014; 26:196-203.

6. Lee $B$, Sandhu S, McArthur G. Cell cycle control as a promising target in melanoma. Curr Opin Oncol 2015; 27:141-150.

7. Blank CU. The perspective of immunotherapy: new molecules and new mechanisms of action in immune modulation. Curr Opin Oncol 2014; 26:204-214.

8. Robert C, Long GV, Brady B, et al. Nivolumab in previously untreated melanoma without BRAF mutation. N Engl J Med 2014. [Epub ahead of print]

9. Wolchok JD, Kluger $\mathrm{H}$, Callahan $\mathrm{MK}$, et al. Nivolumab plus ipilimumab in advanced melanoma. N Engl J Med 2013; 369:122-133.

10. Van Rooij N, van Buuren MM, Philips $D$, et al. Tumor exome analysis reveals neoantigen-specific T-cell reactivity in an ipilimumab-responsive melanoma. J Clin Oncol 2013; 31:e439-e442.

11. Schadendorf $D$, Amonkar MM, Milhem $M$, et al. Functional and symptom impact of trametinib versus chemotherapy in BRAF V600E advanced or metastatic melanoma: quality-of-life analyses of the METRIC study. Ann Oncol 2014; 25:700-706. 